OPEN ACCESS

Edited by:

Claus Ziegler Simonsen, Aarhus University Hospital, Denmark

Reviewed by:

Masahito Kawabori,

Hokkaido University, Japan

Peter Vajkoczy,

Charité Medical University of

Berlin, Germany

Miki Fujimura,

Hokkaido University, Japan

${ }^{*}$ Correspondence:

Yabo Huang

sdfyyhyb@126.com

Specialty section:

This article was submitted to

Stroke,

a section of the journa

Frontiers in Neurology

Received: 20 March 2021

Accepted: 28 June 2021

Published: 23 July 2021

Citation:

Han Q, Yao F, Zhang Z and Huang Y (2021) Evaluation of Revascularization in Different Suzuki Stages of Ischemic Moyamoya Disease by Whole-Brain

CT Perfusion.

Front. Neurol. 12:683224

doi: 10.3389/fneur.2021.683224

\section{Evaluation of Revascularization in Different Suzuki Stages of Ischemic Moyamoya Disease by Whole-Brain CT Perfusion}

\author{
Qingdong Han ${ }^{1}$, Feirong $\mathrm{Yao}^{2}$, Zhengyu Zhang ${ }^{2}$ and Yabo Huang ${ }^{1 *}$ \\ ${ }^{1}$ Department of Neurosurgery, The First Affiliated Hospital of Soochow University, Suzhou, China, ${ }^{2}$ Department of Radiology, \\ The First Affiliated Hospital of Soochow University, Suzhou, China
}

Objective: This study compared the clinical features and hemodynamic characteristics of patients in different Suzuki stages of ischemic moyamoya disease (iMMD) before and after treatment with extracranial-intracranial (EC-IC) bypass surgery combined with encephalo-duro-myo-synangiosis and whole-brain computed tomography perfusion (WB-CTP).

Methods: A total of 126 patients in different Suzuki stages (II, III, IV, and V) of iMMD who underwent bypass surgery from April 2013 to August 2020 were included in this retrospective study. MIStar automatic analysis of Whole brain CT perfusion imaging software (WB-CTP, Apollo Medical Imaging Technology, Melbourne, Australia) was used. The patients also underwent WB-CTP 1 day before and 1 week and 3 months after the surgery. The relationships between hemodynamic parameters in WB-CTP including delay time $(\mathrm{DT})>3 \mathrm{~s}$, relative cerebral blood flow $(\mathrm{rCBF})<30 \%$, mismatch and mismatch ratio, and clinical outcomes were evaluated for different Suzuki stages, with comparisons between early (II and III) and late (IV and V) stages.

Results: Combined bypass surgery was performed in 161 hemispheres of 126 patients with iMMD. Brain volume with DT > $3 \mathrm{~s}$ was decreased 1 week $(51.5 \pm 11.8 \mathrm{ml}, P<$ $0.05)$ and 3 months $(41.5 \pm 10.7 \mathrm{ml}, P<0.05)$ after bypass compared to 1 day before bypass $(104.7 \pm 15.1 \mathrm{ml})$ in early-stage patients. In late-stage patients, the volume was increased 1 week after bypass compared to the preoperative value $(154.3 \pm 14.7$ vs. $118.3 \pm 19.1 \mathrm{ml}, P<0.05)$. Preoperative brain volume with $\mathrm{rCBF}<30 \%$ was lower $(9.8$ \pm 3.9 vs. $33.5 \pm 11.0 \mathrm{ml})$ whereas preoperative mismatch ratio was higher $(11.2 \pm 2.8$ vs. $3.6 \pm 1.6$ ) in early-stage as compared to late-stage patients (both $P<0.05$ ). A higher modified Rankin scale score $(0-1)$ was achieved by early-stage patients than by those in the late stage (93.8 vs. $80.4 \%, P<0.05$ ) at the 3 -month follow-up.

Conclusions: WB-CTP is useful for assessing the effectiveness of combined bypass/revascularization in different Suzuki stages of iMMD. Patients in the early stage of disease with higher preoperative brain volume with DT $>3 \mathrm{~s}$ and mismatch ratio 
show greater improvements in hemodynamic parameters and fewer postoperative complications associated with hemodynamic disturbance following bypass than patients in the late stage. Preoperative mismatch ratio can serve as a marker for assessing the status of collateral circulation in different Suzuki stages of iMMD.

Keywords: moyamoya disease, whole brain CT perfusion, revascularization, hemodynamics, collateral circulation

\section{INTRODUCTION}

Moyamoya disease (MMD) can lead to severe ischemic stroke in adolescent and adult patients (1). Extracranial-intracranial (EC-IC) revascularization by superficial temporal artery-tomiddle cerebral artery (STA-MCA) bypass combined with encephalo-duro-myo-synangiosis (EDAMS) is regarded as an effective treatment for complicated lesions (2-4). However, the consistency between pre- and postoperative hemodynamic characteristics in patients with iMMD in different Suzuki stages, and whether these parameters are related to postoperative complications, is not known.

Whole-brain computed tomography perfusion (WB-CTP) is a useful technique for screening patients with ischemic stroke for treatment with mechanical recanalization or intravenous thrombolysis within a narrow time window $(5,6)$. Although WB-CTP has been applied to MMD to assess the feasibility of revascularization, the relationship between Suzuki stage, WBCTP features, and clinical outcomes has not been reported (7).

In the present study, we investigated the hemodynamic features in WB-CTP of ischemic (i) MMD patients in different Suzuki stages before and after EC-IC revascularization combined with EDAMS, and evaluated the relationship between hemodynamic parameters and clinical outcomes in different Suzuki stages.

\section{METHODS}

\section{Patients}

In this retrospective study, we analyzed 161 hemispheres in 126 patients (61 males and 65 females) with iMMD in different Suzuki stages (II, III, IV, and V) who underwent digital subtraction angiography (DSA) and EC-IC revascularization by STA-MCA bypass combined with EDAMS at the First Affiliated Hospital of Soochow University (Suzhou, China) from April 2013 to August 2020. The surgical and imaging procedures were approved by the ethics committee of the First Affiliated Hospital of Soochow University. All patients provided written, informed consent.

\section{Evaluation of Postoperative Hemodynamics}

MIStar WB-CTP imaging software (Apollo Medical Imaging Technology, Melbourne, Australia) was used to evaluate pre- and postoperative hemodynamics as described in our previous study

Abbreviations: MMD, moyamoya disease; WB-CTP, whole brain CT perfusion; STA, superficial temporal artery; MCA, middle cerebral artery; DT, Delay time; rCBF, relative cerebral blood flow; CTA, computed tomography angiography; CTP, computed tomography perfusion; DSA, digital subtraction angiography; CHS, cerebral hyperperfusion syndrome; TIA, Transient ischemic attack; MRI, magnetic resonance imaging; mRS, modified Rankin scale score.
(8). WB-CTP was performed 1 day before and 1 week and 3 months after bypass. The measured hemodynamic parameters were delay time $(\mathrm{DT})>3 \mathrm{~s}$, relative cerebral blood flow $(\mathrm{rCBF})<$ $30 \%$, and mismatch and mismatch ratios; these were compared between patients with Suzuki stage II, III, IV, and V disease and between early (II and III) and late (IV and V) stages (Figure 1). The mismatch was determined as brain volume with DT $>3 \mathrm{~s}$ minus brain volume rCBF; and mismatch ratio was determined as brain volume with DT $>3$ divided by brain volume with $\mathrm{rCBF}$ $<30 \%$. rCBF was calculated as the absolute values of $\mathrm{CBF}$ in MCA terminal branches divided by the absolute values of $\mathrm{CBF}$ in cerebellar arteries. Two imaging experts blinded to the study analyzed the hemodynamic data.

\section{Treatment of Postoperative Complications}

The following medical interventions were implemented to manage postoperative complications: antioxidant agents, hypotensive drugs, and dehydrating agents for cerebral hyperperfusion syndrome (CHS) (9); and antiplatelet agents for transient ischemic attack (TIA); and antiepileptic drugs and plasma concentration associated with drugs for epilepsy. Patients with minor hemorrhage without mass effect were treated with conservative treatment. The relationships between postoperative complications and hemodynamic parameters before and after bypass were analyzed.

\section{Follow-Up}

Patients were followed up for a maximum of 36 months. Postoperative outcomes were assessed with the modified Rankin scale (mRS) score.

\section{Statistical Analysis}

All variables are presented as mean \pm standard deviations. Categorical variables were analyzed with the chi-squared test, and continuous variables were analyzed with the Student's or paired $t$ test. Data analysis was performed using SPSS v23.0 (SPSS Inc, Chicago, IL, USA). Differences with $P<0.05$ were considered significant.

\section{RESULTS}

\section{Demographic and Clinical Characteristics of the Study Population}

The demographic and clinical characteristics of the study population are shown in Table 1. The mean age of the 126 patients was $46.3 \pm 11.5$ years (range, $15-78$ years). The medical history of the cohort was as follows: hypertension, $n=29$ (23.0\%); diabetes mellitus, $n=24$ (19.0\%); elevated lipids, $n=$ 22 (17.5\%); smoking, $n=25$ (19.8\%); and alcohol abuse, $n=14$ (11.1\%). The number patients in each Suzuki stage was as follows: 


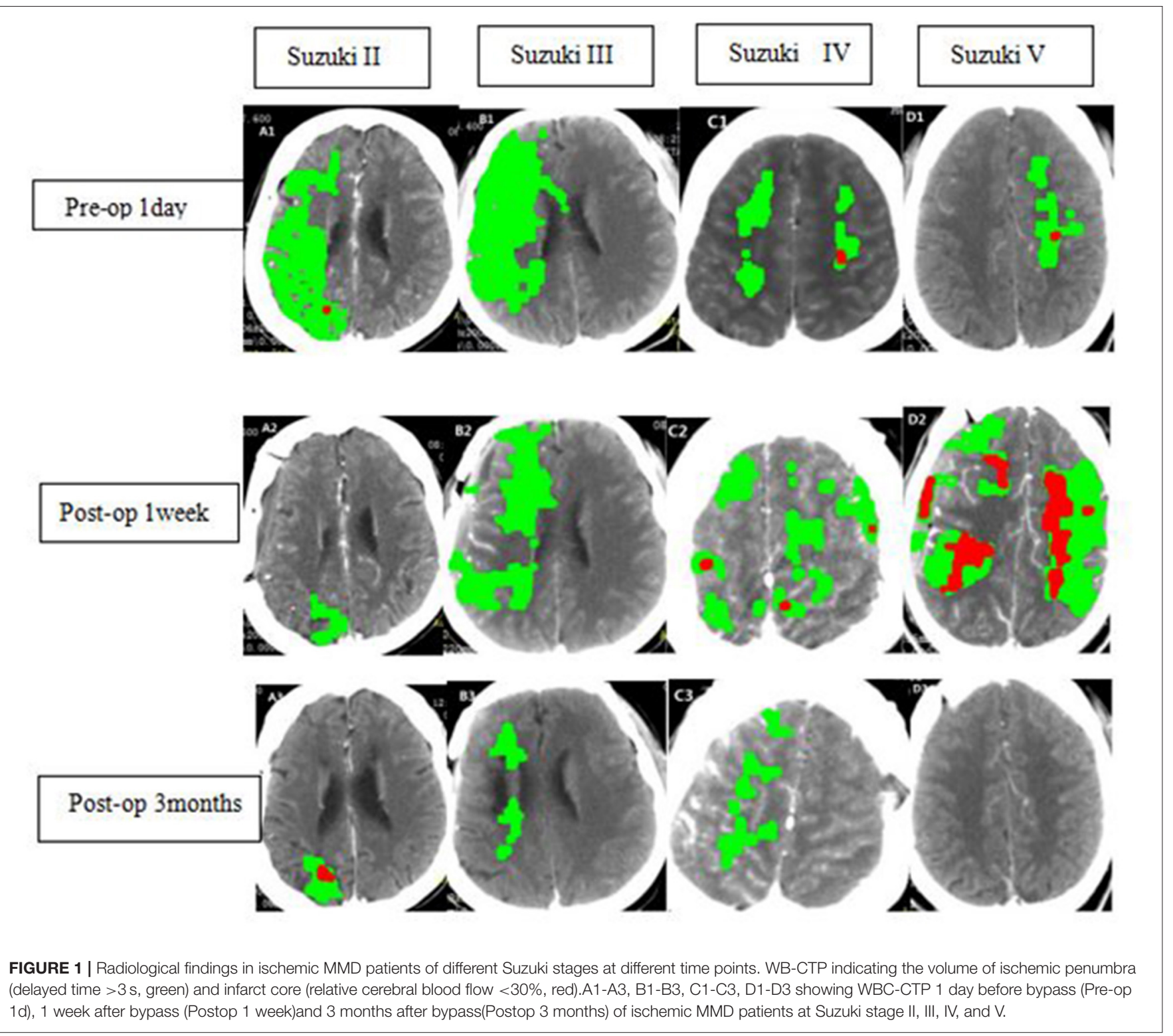

II, $n=11$; III, $n=46$; IV, $n=59$; and V, $n=10$. Preoperative clinical diagnosis included TIA $(n=75,59.5 \%)$ and reversible ischemic neurologic deficit (RIND) $(n=51,40.5 \%)$. A total of 161 hemispheres in the 126 patients were successfully treated by combined bypass/EDAMS. Postoperative complications included CHS $(n=16,12.7 \%)$, TIA $(n=11,8.7 \%)$, epilepsy $(n=10,7.9 \%)$, and intracerebral hemorrhage (ICH) $(n=5,4.0 \%)$.

\section{Hemodynamic Characteristics in Different Suzuki Stages of iMMD Before and After Bypass \\ Hemodynamic Characteristics in Different Suzuki Stages Before and After Bypass}

Patients in different Suzuki stages showed different hemodynamic features in WB-CTP. Postoperative brain volume with DT $>3 \mathrm{~s}$ was significantly decreased 1 week (30.3 \pm $10.2 \mathrm{ml})$ and 3 months $(26.5 \pm 11.5 \mathrm{ml})$ after bypass compared to the preoperative volume $(81.7 \pm 13.6 \mathrm{ml})$ (both $P<0.05)$ in stage II patients (Figure 2). The same trend was observed for patients with stage III disease (1 day before surgery: $115.6 \pm 16.5 \mathrm{ml}$; 1 week after surgery: $62.4 \pm 13.5 \mathrm{ml}$; 3 months after surgery: 49.5 $\pm 10.1 \mathrm{ml}$ ) (both $P<0.05$ ). However, in stage IV patients, brain volume with DT $>3 \mathrm{~s}$ was increased at 1 week after bypass $(160.5$ $\pm 14.1 \mathrm{ml})$ compared to before the surgery $(127.6 \pm 17.7 \mathrm{ml})(P$ $<0.05$ ), although no difference was observed at 3 months (119.7 $\pm 9.8 \mathrm{ml})$. The same was true for patients with stage $\mathrm{V}$ disease ( 1 day before surgery: $101.3 \pm 21.4 \mathrm{ml} ; 1$ week after surgery: 142.2 $\pm 18.6 \mathrm{ml} ; 3$ months after surgery: $93.6 \pm 19.2 \mathrm{ml}$ ).

The postoperative mismatch ratios 1 week $(5.6 \pm 3.4)$ and 3 months (5.5 \pm 3.1$)$ after bypass were significantly lower than the preoperative ratio $(12.7 \pm 5.5, P<0.05)$ in stage II patients (both 
TABLE 1 | Baseline characteristics of 126 ischemic MMD patients.

\begin{tabular}{|c|c|c|c|c|}
\hline Suzuki stage & $\mathrm{II}(11)$ & III(69) & $\operatorname{IV}(36)$ & $V(10)$ \\
\hline \multicolumn{5}{|l|}{ Gender } \\
\hline Male (61) & 4 & 30 & 21 & 6 \\
\hline Female (65) & 7 & 39 & 15 & 4 \\
\hline \multicolumn{5}{|l|}{ Age } \\
\hline$>60$ years $(14)$ & 1 & 7 & 5 & 1 \\
\hline$\leq 60$ years $(112)$ & 10 & 62 & 31 & 9 \\
\hline \multicolumn{5}{|l|}{ Ischemic symptom } \\
\hline TIA (75) & 7 & 38 & 22 & 8 \\
\hline RIND (51) & 4 & 31 & 14 & 2 \\
\hline \multicolumn{5}{|l|}{ Medical history } \\
\hline Hypertension(29) & 2 & 15 & 9 & 3 \\
\hline Smoking (25) & 4 & 10 & 9 & 2 \\
\hline DM (24) & 1 & 13 & 8 & 2 \\
\hline High lipid (22) & 2 & 11 & 7 & 2 \\
\hline Alcohol abusing (14) & 1 & 7 & 9 & 2 \\
\hline \multicolumn{5}{|l|}{ Pre-operative mRS Scale } \\
\hline $0-1(86)$ & 9 & 47 & 23 & 7 \\
\hline $2-3(40)$ & 2 & 22 & 13 & 3 \\
\hline \multicolumn{5}{|l|}{ Revascularization side } \\
\hline Left side (50) & 6 & 29 & 12 & 3 \\
\hline Right side (51) & 3 & 26 & 16 & 6 \\
\hline Left and Right sides(25) & 2 & 14 & 8 & 1 \\
\hline \multicolumn{5}{|l|}{ Post-operative complication } \\
\hline CHS (16) & 1 & 6 & 7 & 2 \\
\hline TIA (11) & 1 & 5 & 4 & 1 \\
\hline Epilepsy(10) & 0 & 2 & 2 & 1 \\
\hline Intra-cerebral hemorrhage (5) & 0 & 2 & 2 & 1 \\
\hline \multicolumn{5}{|l|}{ Post-operative mRS scale } \\
\hline $0-1(112)$ & 10 & 65 & 28 & 9 \\
\hline $2-3(14)$ & 1 & 4 & 8 & 1 \\
\hline
\end{tabular}

TIA, Transient ischemic attack; RIND, Reversible ischemic neurological deficit. DM, Diabetes mellitus; CHS, Cerebral hyperperfusion syndrome; mRS, Modified Rankin Scale.

$P<0.05$ ) (Figure 3 and Table 2). For patients in Suzuki stage III, a significant decrease compared to the preoperative mismatch ratio $(10.8 \pm 4.2)$ was observed only at the 1-week follow-up $(4.1 \pm 2.5)(P<0.05)$, with no difference at 3 months $(3.4 \pm$ 1.4). Patients in stages IV and V showed no differences between preoperative and 1-week or 3-month postoperative mismatch ratios (stage IV: $3.7 \pm 1.5$ vs. $3.9 \pm 1.2$ vs. $3.3 \pm 1.9$; stage V: 3.4 \pm 1.6 vs. $5.4 \pm 2.7$ vs. $3.6 \pm 1.5$ ) (both $P>0.05$ ).

\section{Hemodynamic Characteristics in Early vs. Late Suzuki Stage Before and After Bypass}

Hemodynamics characteristics of early-stage (II and III) and late-stage (IV and V) iMMD patients differed significantly. Postoperative brain volume with DT $>3 \mathrm{~s}$ was markedly lower 1 week $(51.5 \pm 11.8 \mathrm{ml})$ and 3 months $(41.5 \pm 10.7 \mathrm{ml})$ after bypass compared to the preoperative volume $(104.7 \pm 15.1 \mathrm{ml})$ (both $P<0.05$ ) in early-stage patients (Figure 4 and Table 3 ). In contrast, in late-stage patients the volume was significantly higher 1 week after as compared to 1 day before the surgery (154.3 \pm 14.7 vs. $118.3 \pm 19.1 \mathrm{ml} ; P<0.05)$, whereas no significant difference relative to the preoperative value was observed at the
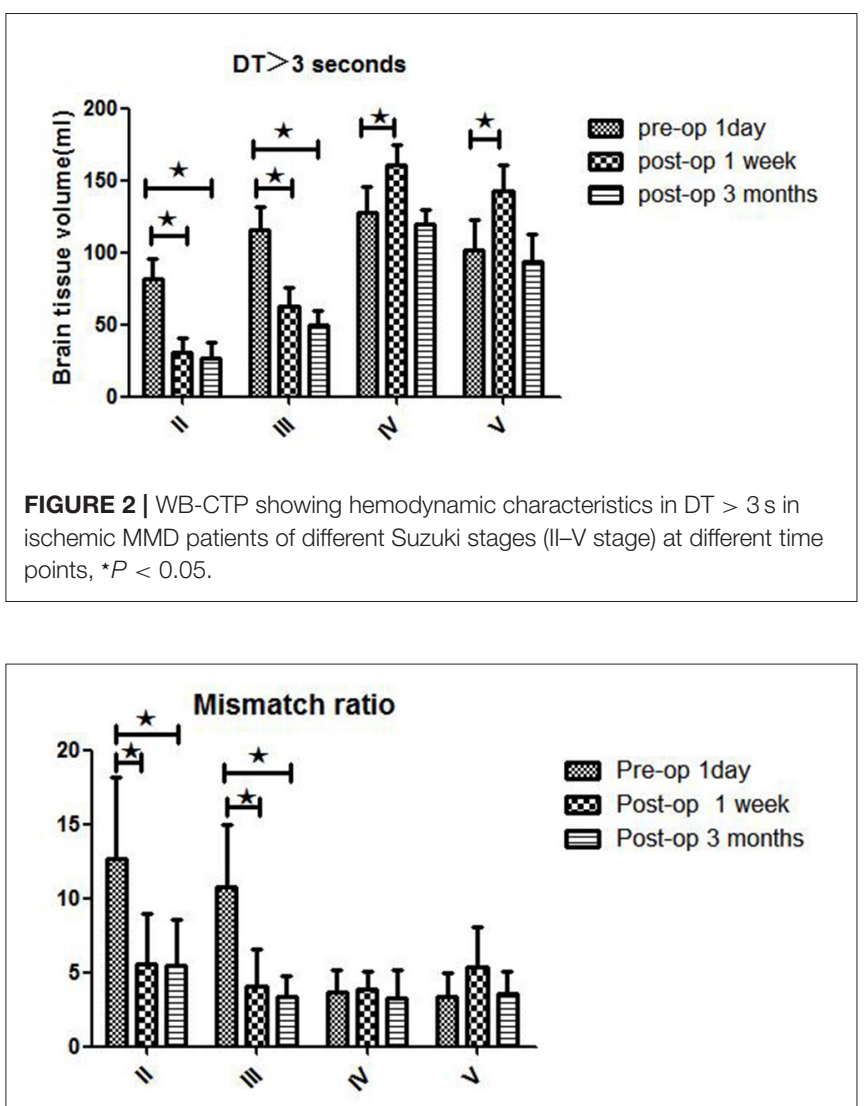

FIGURE 3 | WB-CTP showing hemodynamic characteristics in DT > $3 \mathrm{~s}$ in ischemic MMD patients of different Suzuki stages (early and later stage) at different time points. Early stage: in Suzuki stage II and III Later stage:in Suzuki stage IV and $\mathrm{V}$. ${ }^{\star} P<0.05$.

3-month follow-up (109.5 $\pm 16.3 \mathrm{ml} ; P>0.05)$. Early- and latestage patients showed significant differences in mismatch ratio 1 day before bypass $(11.2 \pm 2.8$ vs. $3.6 \pm 1.6, P<0.05)$, but no significant differences at 1 week $(4.5 \pm 2.1$ vs. $4.3 \pm 1.9, P>0.05)$ and 3 months $(4.2 \pm 1.7$ vs. $3.4 \pm 1.5, P>0.05)$ after bypass (Figure 5).

\section{Relationship Between Hemodynamic Findings and Postoperative Complications in Different Suzuki Stages in iMMD}

The rates of postoperative complications in different Suzuki stages were as follows: stage II, 2/11 (18.2\%); stage III, 17/69 (24.6\%); stage IV, 18/36 (50\%); stage V, 5/10 (50\%) (Table 1). The 4 major types of postoperative complication occurred at higher rates in late-stage as compared to early-stage patients, including CHS (19.6 vs. $8.6 \%)$, TIA (10.9 vs. $7.5 \%$ ), epilepsy (13.0 vs. $5.0 \%)$, and $\mathrm{ICH}$ (6.5 vs. $2.5 \%)$.

Preoperative brain volume with $\mathrm{rCBF}<30 \%$ was lower in patients in the early stage $(9.8 \pm 3.9 \mathrm{ml})$ than in patients in the late stage $(33.5 \pm 11.0 \mathrm{ml})$ of iMMD $(P<0.05)$ (Table 3$)$. Accordingly, preoperative mismatch ratio was higher in the early stage as compared to the late stage of disease (11.2 \pm 2.8 vs. $3.6 \pm$ 1.6; $P<0.05)$. Of the 80 patients in the early stage, $56(70.0 \%)$ 


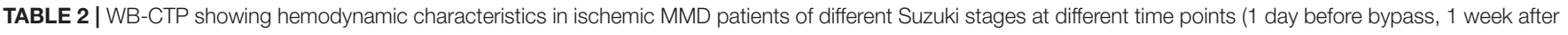
bypass and 3 months following bypass).

\begin{tabular}{|c|c|c|c|c|c|c|c|c|c|}
\hline & \multicolumn{3}{|c|}{ Pre-operative 1day } & \multicolumn{3}{|c|}{ Post-operative 1 week } & \multicolumn{3}{|c|}{ Post-operative 3months } \\
\hline & DT>3s (ml) & $\mathrm{rCBF}<30 \%(\mathrm{ml})$ & ${ }^{*} \mathrm{MR}(\mathrm{ml})$ & $\mathrm{DT}>3 \mathrm{~s}(\mathrm{ml})$ & $\mathrm{rCBF}<30 \%(\mathrm{ml})$ & ${ }^{*} \mathrm{MR}(\mathrm{ml})$ & $\mathrm{DT}>3 \mathrm{~s}(\mathrm{ml})$ & $\mathrm{rCBF}<30 \%(\mathrm{ml})$ & ${ }^{*} \mathrm{MR}(\mathrm{ml})$ \\
\hline ॥ & $81.7 \pm 13.6$ & $7.0 \pm 4.1$ & $12.7 \pm 5.5$ & $30.3 \pm 10.2$ & $6.1 \pm 2.6$ & $5.6 \pm 3.4$ & $26.5 \pm 11.5$ & $5.4 \pm 3.3$ & $5.5 \pm 3.1$ \\
\hline III & $115.6 \pm 16.5$ & $11.2 \pm 3.5$ & $10.8 \pm 4.2$ & $62.4 \pm 13.5$ & $15.8 \pm 5.7$ & $4.1 \pm 2.5$ & $49.5 \pm 10.1$ & $14.5 \pm 6.7$ & $3.4 \pm 1.4$ \\
\hline IV & $127.6 \pm 17.7$ & $34.9 \pm 11$ & $3.7 \pm 1.5$ & $160.5 \pm 14.1$ & $41.2 \pm 0.9$ & $3.9 \pm 1.2$ & $119.7 \pm 9.8$ & $36.7 \pm 13.7$ & $3.3 \pm 1.9$ \\
\hline V & $101.3 \pm 21.4$ & $31.7 \pm 10.5$ & $3.4 \pm 1.6$ & $142.2 \pm 18.6$ & $26.4 \pm 1.7$ & $5.4 \pm 2.7$ & $93.6 \pm 19.2$ & $25.5 \pm 9.7$ & $3.6 \pm 1.5$ \\
\hline
\end{tabular}

${ }^{\star} M R$, mismatch ratio (volume of DT $>3$ divide volume of $r C B F<30 \%$ ).

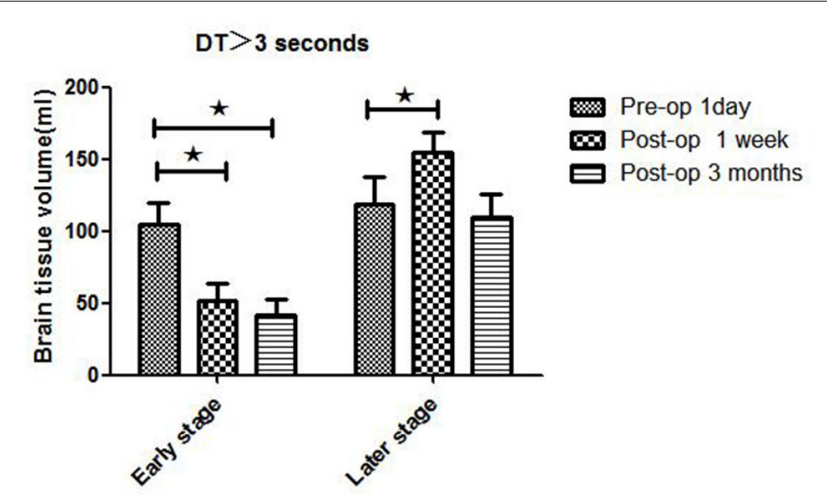

FIGURE 4 | WB-CTP showing hemodynamic characteristics in mismatch ratio in ischemic MMD patients of different Suzuki stages (II-V stage) at different time points. Early stage: in Suzuki stage II and III Later stage:in Suzuki stage IV and $\mathrm{V}$. ${ }^{\star} \mathrm{MR}$, mismatch ratio (volume of DT $>3$ divide volume of $\mathrm{rCBF}<30 \%$ ), ${ }^{\star} P<0.05$.

had a preoperative mRS score of $0-1$; the number of patients with scores in this range increased to 75 (93.8\%) at 3 months after surgery. Among the 46 patients in the late stage, 30 (65.2\%) had an mRS score of $0-1$ before the surgery and the number increased to $37(80.4 \%)$ at 3 months after bypass; the difference between early- and late-stage patients at the 3-month follow-up was statistically significant $(P<0.05)$.

\section{DISCUSSION}

MMD is a type of cerebrovascular lesion whose etiology is not fully understood. Given the occlusion of intracranial arteries, establishing collateral circulation through EC-IC bypass can increase cerebral blood flow, thereby preventing ischemic stroke or hemorrhage (10-13). However, in order to ensure good clinical outcomes with this approach, it is important to understand the hemodynamic characteristics of iMMD patients in different Suzuki stages and postoperative complications associated with hemodynamic variances.

The iMMD patients in different Suzuki stages in our study showed differences in hemodynamic features in WB-CTP. A higher mismatch ratio and lower brain volume with $\mathrm{rCBF}<$ $30 \%$ are known to be related to better collateral circulation, which has been used to guide the treatment of embolism in ischemic stroke (14). In the present study, the hemodynamic parameters of early Suzuki stage (II and III) patients markedly improved after bypass surgery. However, the same was not true

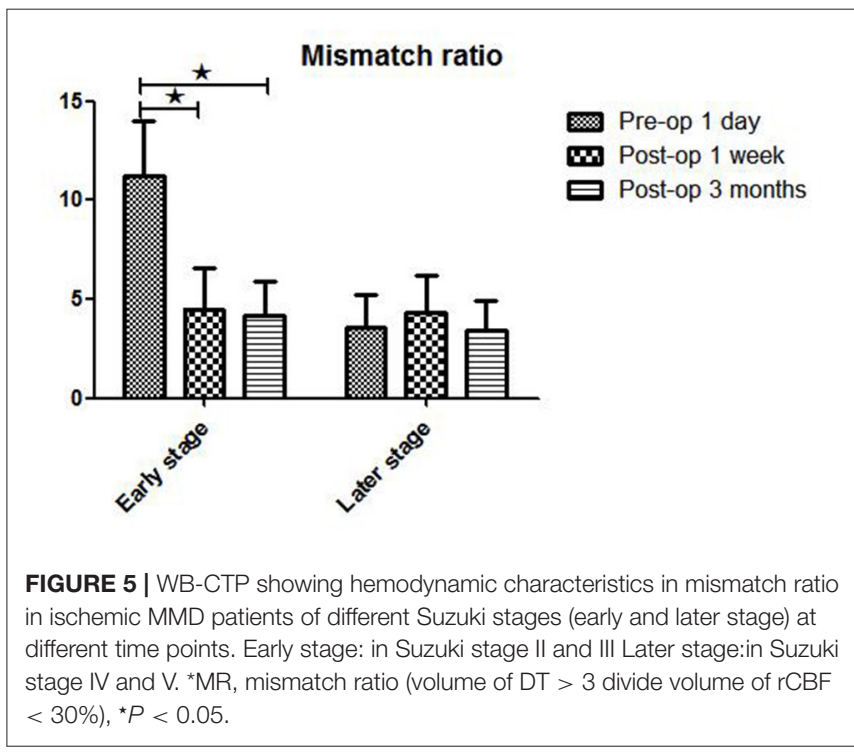

of patients in the late stages (IV and V) of disease, who showed increased volumes of both the ischemic core and penumbra. The preoperative mismatch ratio was also lower in late-stage MMD patients, indicating an insufficiency of collateral vessels. Thus, preoperative mismatch ratio can be considered as a marker for the status of collateral circulation in different Suzuki stages of iMMD. Additionally, the possibility of a postoperative decrease in blood vessel formation following EC-IC revascularization and cerebral cortex perfusion should be noted (7). In our study, the postoperative mismatch ratio was decreased in both early- and late-stage patients following bypass, which is in accordance with previous reports (7).

The observed changes in collateral circulation and hemodynamics following bypass surgery were related to postoperative complications. Mismatch in the cerebral cortex is thought to reflect a salvageable brain area after ischemic stroke (5). EC-IC bypass can have a marked effect on collateral circulation, inducing hemodynamic disturbances such as cerebral hyperperfusion syndome and postoperative watershed shift ischemia (15). It has been reported that micro embolism was related to MMD progression and infarct onset (10). One study proposed that collateral circulation in MMD be classified based on whether the origin is the external or internal carotid or vertebral artery, as patients with collaterals originating from the external carotid artery usually had late-stage disease and were at a higher risk of infarction (16). So we protect 
TABLE 3 | WB-CTP showing hemodynamic characteristics in ischemic MMD patients of different Suzuki stages (in early stage and later stage) at different time points.

\begin{tabular}{|c|c|c|c|c|c|c|c|c|c|}
\hline & \multicolumn{3}{|c|}{ Pre-operative 1day } & \multicolumn{3}{|c|}{ Post-operative 1 week } & \multicolumn{3}{|c|}{ Post-operative 3months } \\
\hline & $\mathrm{DT}>3 \mathrm{~s}(\mathrm{ml})$ & rCBF $<30 \%(\mathrm{ml})$ & *MR (ml) & $\mathrm{DT}>3 \mathrm{~s}(\mathrm{ml})$ & $r C B F<30 \%(m l)$ & *MR (ml) & $\mathrm{DT}>3 \mathrm{~s}(\mathrm{ml})$ & $\mathrm{rCBF}<30 \%(\mathrm{ml})$ & *MR (ml) \\
\hline $\begin{array}{l}\text { Early } \\
\text { stage }\end{array}$ & $104.7 \pm 15.1$ & $9.8 \pm 3.9$ & $11.2 \pm 2.8$ & $51.5 \pm 11.8$ & $11.6 \pm 4.5$ & $4.5 \pm 2.1$ & $41.5 \pm 10.7$ & $11.8 \pm 5.5$ & $4.2 \pm 1.7$ \\
\hline $\begin{array}{l}\text { Later } \\
\text { stage }\end{array}$ & $118.3 \pm 19.1$ & $33.5 \pm 11.0$ & $3.6 \pm 1.6$ & $154.3 \pm 14.7$ & $35.7 \pm 11.2$ & $4.3 \pm 1.9$ & $3.4 \pm 1.5$ & $109.5 \pm 16.3$ & $32.4 \pm 11.1$ \\
\hline
\end{tabular}

Early stage: in Suzuki stage II and III Later stage: in Suzuki stage IV and V.

${ }^{\star} \mathrm{MR}$, mismatch ratio (volume of DT $>3$ divide volume of $\mathrm{rCBF}<30 \%$ ).

trans-dural anastomosis, such as middle meningeal arteries, during craniotomy especially in patients with late Suzuki's stage. Previous report indicated the significant association of the risk of cerebral hyperperfusion with the higher Suzuki's angiographic stage (17).

Our study showed that the rate and severity of postoperative complications (CHS, TIA, epilepsy, and ICH) in early- vs. late-stage iMMD patients differed significantly. CTP provides a method for quantitatively evaluating cerebral perfusion; a discrepancy between cerebral blood flow and ischemic presentation indicates that both cerebrovascular reserve and collateral circulation are responsible for hemodynamic disturbances (18). In our study, the infarct core $(\mathrm{rCBF}<30 \%)$ and mismatch ratio differed markedly between early- and late-stage patients, implying poor collateral circulation and impaired auto-regulatory function of the cerebral vasculature in the latter group. Dramatic and sudden hemodynamic changes following bypass can result in excessive blood flow, leading to CHS or ICH (19). Postoperative watershed ischemia has been attributed to competition between high blood flow from the STA and intrinsically low or retrograde flow in the MCA (20).

Other imaging findings can facilitate the assessment of hemodynamic changes during EC-IC bypass in the treatment of iMMD. $\mathrm{CO}_{2}$-triggered blood oxygen level-dependent functional magnetic resonance imaging (MRI) can reveal regional cerebrovascular reserve or reactivity in MMD patients (21), but variations in breath holding across patients can influence the results. As a non-invasive tool, duplex ultrasonography has been used to examine collateral circulation pre- and postoperatively, allowing neurosurgeons to predict clinical outcomes $(22,23)$, although this may be susceptible to inter-observer variations. Image-based computational fluid dynamics in CTP can provide information on graft blood flow, but the distal portion of the STA graft is difficult to visualize, especially in pediatric patients (24). The ivy sign in the ipsilateral hemisphere in fluid-attenuated inversion recovery MRI before and after revascularization was shown to be an indicator of hyperperfusion (25). Finally, quantitative single-photon emission computed tomography (SPECT) can be used to monitor postoperative hemodynamic disturbances (20).

There were some limitations to our study that warrant attention. Firstly, we analyzed data from a single center, which could limit the generalizability of our findings. Secondly, only patients with stage II-V iMMD were included in the analysis; additional studies are needed to determine the hemodynamic profiles of patients in other stages (i.e., I and VI). Thirdly, our analyses were based on radiologic findings but data obtained from other imaging modalities and examinations should be considered in the evaluation of postoperative hemodynamic status.

\section{CONCLUSION}

WB-CTP is a useful method for evaluating the effectiveness of combined revascularization in the treatment of iMMD. Patients in the early stages of disease (Suzuki stages II and III) can achieve greater improvements in hemodynamic parameters with fewer hemodynamic complications following EC-IC bypass surgery than those in the late stages (Suzuki stages IV and V). Preoperative mismatch ratio can serve as a marker for assessing the status of collateral circulation in different Suzuki stages of iMMD.

\section{DATA AVAILABILITY STATEMENT}

The raw data supporting the conclusions of this article will be made available by the authors, without undue reservation.

\section{ETHICS STATEMENT}

The studies involving human participants were reviewed and approved by the Ethics Committee of the First Hospital of Soochow University. Written informed consent to participate in this study was provided by the participants' legal guardian/next of kin.

\section{AUTHOR CONTRIBUTIONS}

$\mathrm{YH}$ and QH: design of the study, writing — review and editing, and supervision. FY and QH: data resources. FY and ZZ: imaging analysis. QH and YH: writing-original draft. QH and FY: statistical analysis. All authors contributed to the article and approved the submitted version.

\section{ACKNOWLEDGMENTS}

We thank the patients for their participation and our staff members for assistance. 


\section{REFERENCES}

1. Kim WH, Kim SD, Nam MH, Jung JM, Jin SW, Ha SK, et al. Posterior circulation involvement and collateral flow pattern in moyamoya disease with the RNF213 polymorphism. Childs Nerv Syst. (2019) 35:30914. doi: 10.1007/s00381-018-3985-5

2. Nielsen TH, Abhinav K, Sussman ES, Han SS, Weng Y, Bell-Stephens T, et al. Direct versus indirect bypass procedure for the treatment of ischemic moyamoya disease: results of an individualized selection strategy. J Neurosurg. (2020) 12:1-12. doi: 10.3171/2020.3.JNS192847

3. Lei Y, Li YJ, Guo QH, Liu XD, Liu Z, Ni W, et al. Postoperative executive function in adult moyamoya disease: a preliminary study of its functional anatomy and behavioral correlates. J Neurosurg. (2017) 126:52736. doi: $10.3171 / 2015.12$.JNS151499

4. Tokairin K, Kazumata K, Uchino H, Ito M, Ono K, Tatezawa R, et al. Postoperative intracerebral hemorrhage after combined revascularization surgery in moyamoya disease: profiles and clinical associations. World Neurosurg. (2018) 120:e593-600. doi: 10.1016/j.wneu.2018.08.132

5. Rudilosso S, Urra X, San Roman L, Laredo C, Lopez-Rueda A, Amaro $\mathrm{S}$, et al. Perfusion deficits and mismatch in patients with acute lacunar infarcts studied with whole-brain CT perfusion. AJNR. (2015) 36:140712. doi: 10.3174/ajnr.A4303

6. Morelli $\mathrm{Nj}$, Rota E, Colombi D, Marchesi G, Villaggi E, Giordano $\mathrm{C}$, et al. Goliath and the ant: whole-brain CT perfusion against 16slice CT angiography in stroke imaging. J Neuroradiol. (2019) 46:398400. doi: $10.1016 /$ j.neurad.2019.02.012

7. Zhang J, Wang J, Geng D, Li Y, Song D, Gu Y. Whole-brain CT perfusion and $\mathrm{CT}$ angiography assessment of Moyamoya disease before and after surgical revascularization: preliminary study with 256-slice CT. PLoS ONE. (2013) 8:e57595. doi: 10.1371/journal.pone.0057595

8. Yao F, Han Q, Zhang Z, Huang Y. Assessment of transient cerebral hemodynamic disturbance via whole-brain computed tomography perfusion after extracranial-intracranial bypass in ischemic moyamoya disease. World Neurosurg. (2021) 146:e112-121. doi: 10.1016/j.wneu.2020.10.046

9. Zhang J, Li S, Fujimura M, Lau TY, Wu X, Hu M, et al. Hemodynamic analysis of the recipient parasylvian cortical arteries for predicting postoperative hyperperfusion during STA-MCA bypass in adult patients with moyamoya disease. J Neurosurg. (2021) 134:17-24. doi: 10.3171/2019.10.JNS191207

10. Kim DY, Son JP, Yeon JY, Kim GM, Kim JS, Hong SC, et al. Infarct pattern and collateral status in adult moyamoya disease: a multimodal magnetic resonance imaging study. Stroke. (2017) 48:1116. doi: 10.1161/STROKEAHA.116.014529

11. Suzuki J, Takaku A. Cerebrovascular "moyamoya" disease. Disease showing abnormal net-like vessels in base of brain. Arch Neurol. (1969) 20:28899. doi: 10.1001/archneur.1969.00480090076012

12. Fujimura M, Tominaga T. Current status of revascularization surgery for Moyamoya disease: special consideration for its 'internal carotid-external carotid (IC-EC) conversion' as the physiological reorganization system. Tohoku J Exp Med. (2015) 236:45-53. doi: 10.1620/tjem.236.45

13. Zhu F, Qian Y, Xu B, Gu Y, Karunanithi K, Zhu W, et al. Quantitative assessment of changes in hemodynamics of the internal carotid artery after bypass surgery for moyamoya disease. J Neurosurg. (2018) 129:67783. doi: 10.3171/2017.5.JNS163112

14. Lu SS, Zhang X, Xu XQ, Cao YZ, Zhao LB, Liu QH, et al. Comparison of CT angiography collaterals for predicting target perfusion profile and clinical outcome in patients with acute ischemic stroke. Eur Radiol. (2019) 29:4922-9. doi: 10.1007/s00330-019-06027-9

15. Han Q, Huang Y. Quantitative analysis of revascularization in ischemic moyamoya disease via whole-brain computed tomography perfusion: a retrospective single-center study. Medicine. (2020) 99:e19168. doi: 10.1097/MD.00000000000 19168
16. Zhao M, Zhang D, Wang S, Zhang Y, Deng X, Zhao J. The collateral circulation in moyamoya disease: a single-center experience in 140 pediatric patients. Pediatr Neurol. (2017) 77:78-83. doi: 10.1016/j.pediatrneurol.2017.08.016

17. Katsuki M, Fujimura M, Tashiro R, Tomata Y, Nishizawab T, Tominaga T. Pre-operative higher hematocrit and lower total protein levels are independent risk factors for cerebral hyperperfusion syndrome after superficial temporal artery-middle cerebral artery anastomosis with pial synangiosis in adult moyamoya disease patients-case-control study. Neurosurg Rev. (2020). doi: 10.1007/s10143-020-01395-z. [Epub ahead of print].

18. Sasagawa A, Mikami T, Hirano T, Akiyama Y, Mikuni N. Characteristics of cerebral hemodynamics assessed by CT perfusion in moyamoya disease. J Clin Neurosci. (2018) 47:183-9. doi: 10.1016/j.jocn.2017.09.020

19. Ishikawa T, Yamaguchi K, Kawashima A, Funatsu T, Eguchi S, Matsuoka G, et al. Predicting the occurrence of hemorrhagic cerebral hyperperfusion syndrome using regional cerebral blood flow after direct bypass surgery in patients with moyamoya disease. World Neurosurg. (2018) 119:e7506. doi: 10.1016/j.wneu.2018.07.258

20. Tu XK, Fujimura M, Rashad S, Mugikura S, Sakata H, Niizuma $\mathrm{K}$, et al. Uneven cerebral hemodynamic change as a cause of neurological deterioration in the acute stage after direct revascularization for moyamoya disease: cerebral hyperperfusion and remote ischemia caused by the 'watershed shift.' Neurosurg Rev. (2017) 40:507-12. doi: 10.1007/s10143-017-0845-9

21. Hauser TK, Seeger A, Bender B, Klose U, Thurow J, Ernemann U, et al. Hypercapnic BOLD MRI compared to H2(15)O PET/CT for the hemodynamic evaluation of patients with moyamoya disease. Neuroimage Clin. (2019) 22:101713. doi: 10.1016/j.nicl.2019.101713

22. Yeh SJ, Tang SC, Tsai LK, Lee CW, Chen YF, Liu HM, et al. Color doppler ultrasonography as an alternative tool for postoperative evaluation of collaterals after indirect revascularization surgery in Moyamoya disease. PLoS ONE. (2017) 12:e0188948. doi: 10.1371/journal.pone.0188948

23. Wang Y, Chen L, Wang Y, Pan H, Wan Y, Xu B, et al. Hemodynamic study with duplex ultrasonography on combined (direct/indirect) revascularization in adult moyamoya disease. J Stroke Cerebrovasc Dis. (2014) 23:25739. doi: 10.1016/j.jstrokecerebrovasdis.2014.05.024

24. Kuribara T, Mikami T, Komatsu K, Kimura Y, Kim S, Miyata $\mathrm{K}$, et al. Preoperatively estimated graft flow rate contributes to the improvement of hemodynamics in revascularization for Moyamoya disease. J Stroke Cerebrovasc Dis. (2021) 30:105450. doi: 10.1016/j.jstrokecerebrovasdis.2020.105450

25. Horie N, Morikawa M, Morofuji Y, Hiu T, Izumo T, Hayashi K, et al. De novo ivy sign indicates postoperative hyperperfusion in moyamoya disease. Stroke. (2014) 45:1488-91. doi: 10.1161/STROKEAHA.114.004755

Conflict of Interest: The authors declare that the research was conducted in the absence of any commercial or financial relationships that could be construed as a potential conflict of interest.

Publisher's Note: All claims expressed in this article are solely those of the authors and do not necessarily represent those of their affiliated organizations, or those of the publisher, the editors and the reviewers. Any product that may be evaluated in this article, or claim that may be made by its manufacturer, is not guaranteed or endorsed by the publisher.

Copyright (๑ 2021 Han, Yao, Zhang and Huang. This is an open-access article distributed under the terms of the Creative Commons Attribution License (CC BY). The use, distribution or reproduction in other forums is permitted, provided the original author(s) and the copyright owner(s) are credited and that the original publication in this journal is cited, in accordance with accepted academic practice. No use, distribution or reproduction is permitted which does not comply with these terms. 